\title{
Cavity Liner
}

National Cancer Institute

\section{Source}

National Cancer Institute. Cavity Liner. NCI Thesaurus. Code C62126.

A material placed between the restoration and the tooth surface. 